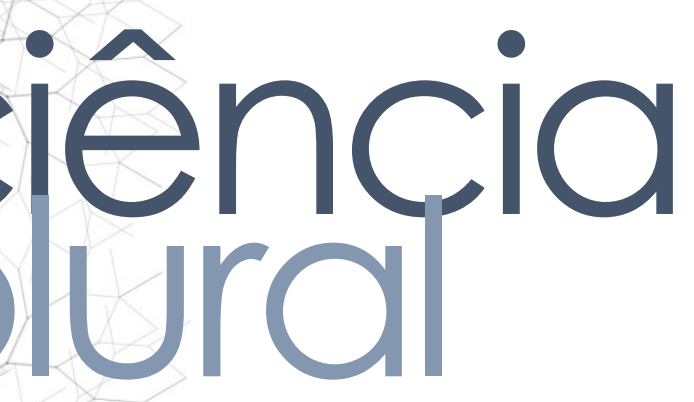

\title{
DESAFIOS E ADAPTAÇÕES DA EXTENSÃO UNIVERSITÁRIA EM TEMPOS DE PANDEMIA: RELATO DE EXPERIÊNCIA
}

Challenges and adaptations of university extension in pandemic times: experience report

Retos y adaptaciones de la Extensión universitaria en tiempos de pandemia: informe de experiencia

Ruan Kaio Silva Nunes • Acadêmico e extensionista do curso de Nutrição da Universidade do Vale do Itajaí-UNIVALI • E-mail: ruan-kaiio@hotmail.com

Gabrielle Augustha dos Santos Maciel • Acadêmico e extensionista do curso de Nutrição da UNIVALI • E-mail: gabiaugustha.mac@gmail.com

Elisabeth Barth Almeida • Nutricionista, • Mestre em Turismo e Hotelaria • Docente e pesquisadora do curso de Nutrição da UNIVALI • E-mail: bethbarth@univali.com

Mariana Reiser Guedes • Mestranda em Saúde e Gestão do Trabalho pela UNIVALI - Docente do curso de Nutrição da UNIVALI• E-mail: marianaguedes@univali.com

Rosana Henn • Nutricionista • Mestre em Ciência dos Alimentos • Docente e pesquisadora do curso de Nutrição da UNIVALI • E-mail: rhenn@univali.br

Autor responsável pela correspondência:

Ruan Kaio Silva Nunes• E-mail: ruan-kaiio@hotmail.com 


\section{ciêncíncia
puúl}

\section{RESUMO}

Introdução: A crise pelo novo Coronavírus tem influenciado diversos setores, dentre eles o da educação superior, pois as atividades realizadas dentro da universidade estão, desde março de 2020, sendo remanejadas e readaptadas para o modelo on-line, dentre elas a extensão universitária. Objetivo: $O$ presente artigo tem o objetivo de descrever a vivência de um projeto de extensão universitária desenvolvido para estimular a adoção de hábitos alimentares saudáveis e que migrou suas atividades para o ambiente virtual, mostrando como foram os desafios e adaptações nesse novo cenário global. Metodologia: Trata-se de um estudo descritivo, do tipo relato de experiência. As atividades adaptadas à nova realidade que serão aqui mencionadas ocorreram no primeiro semestre de 2020, iniciando no mês de maio e finalizando em julho. As postagens para as redes sociais Facebook ${ }^{\circledR}$ e Instagram $^{\circledR}$ foram realizadas uma vez por semana, por dois acadêmicos do curso de Nutrição e bolsistas do projeto, tendo sempre o auxílio das professoras responsáveis. Resultados: A utilização das redes sociais Facebook $^{\circledR}$ e Instagram $^{\circledR}$ ajudaram nosso projeto a desempenhar suas atividades de forma remota e obter um alcance maior de pessoas beneficiadas com as publicações. Conclusão: Apesar das dificuldades iniciais de planejamento de cronograma, temas abordados e o alcance do público-alvo, conseguimos reestruturar bem o projeto ao novo formato. Conseguimos perceber, com a elaboração dos materiais para as publicações e os retornos recebidos, que tivemos a capacidade de influenciar positivamente a vida das pessoas, buscando trazer assuntos pertinentes e com embasamento científico, em linguagem de fácil entendimento, para assim, mesmo que remotamente, tentar melhorar a qualidade de vida da população. Reforçamos a importância das redes sociais para o aumento do número de indivíduos beneficiados pelo projeto de extensão durante o período pandêmico.

Palavras-Chave: Relações Comunidade-Instituição; Pandemias; Prevenção de Doenças; Rede Social.

\section{ABSTRACT}

Introduction: The crisis with the new Coronavirus has influenced several sectors, including higher education, since the activities carried out within the university are, since March 2020, being relocated and readapted for the online model, among them the university extension. Objective: This article aims to describe the experience of a university extension project developed to encourage the adoption of healthy eating habits and which migrated its activities to the virtual environment, showing how the challenges and adaptations were in this new global scenario. Methodology: This is a descriptive, experience report type study. The activities adapted to the new reality that will be mentioned here took place in the first half of 2020, starting in May and ending July. The posts for the social networks Facebook $\AA$ and Instagram $\AA$ were made once a eek, by two students of the Nutrition course and scholarship students of the project, ways with the help of the responsible teachers. Results: The use of the social tworks Facebook ${ }^{\circledR}$ and Instagram ${ }^{\circledR}$ helped our project to carry out its activities notely and obtain a greater reach of people who benefit from the publications. clusion: Despite the initial difficulties in planning the schedule, topics covered 
and the reach of the target audience, we managed to restructure the project well to the new format. We were able to realize, with the preparation of the materials for the publications and the feedback received, that we had the ability to positively influence people's lives, seeking to bring pertinent and scientifically based matters, in language that is easy to understand, so that even remotely try to improve the quality of life of the population. We reinforce the importance of social networks for increasing the number of individuals benefited by the extension project during the pandemic period.

Keywords: Community-Institutional Relations; Pandemics; Disease Prevention; Social Networking.

\section{RESUMEN}

Introducción: La crisis con el nuevo Coronavirus ha influido en varios sectores, entre ellos la educación superior, ya que las actividades que se llevan a cabo dentro de la universidad están, desde marzo de 2020, siendo reubicadas y readaptadas para el modelo online, entre ellos la extensión universitaria. Objetivo: Este artículo tiene como objetivo describir la experiencia de un proyecto de extensión universitaria desarrollado para incentivar la adopción de hábitos alimentarios saludables y que migró sus actividades al entorno virtual, mostrando cómo fueron los desafíos y adaptaciones en este nuevo escenario global. Metodología: Se trata de un estudio descriptivo tipo relato de experiencia. Las actividades adaptadas a la nueva realidad que aquí mencionaremos tuvieron lugar en el primer semestre de 2020, comenzando en mayo y finalizando en julio. Las publicaciones para las redes sociales Facebook® e Instagram ${ }^{\circledR}$ fueron realizada una vez a la semana, por dos alumnos del curso de Nutrición y becarios del proyecto, siempre con la ayuda de los profesores responsables. Resultados: El uso de las redes sociales Facebook® e Instagram $₫$ ayudó a nuestro proyecto a realizar sus actividades de forma remota y obtener un mayor alcance de personas que se benefician de las publicaciones. Conclusión: A pesar de las dificultades iniciales en la planificación del cronograma, los temas tratados y el alcance de la audiencia objetivo, logramos reestructurar bien el proyecto al nuevo formato. Pudimos darnos cuenta, con la elaboración de los materiales para las publicaciones y la retroalimentación recibida, que teníamos la capacidad de influir positivamente en la vida de las personas, buscando traer asuntos pertinentes y con base científica, en un lenguaje fácil de entender, de manera que, incluso de forma remota, tratar de mejorar la calidad de vida de la población. Reforzamos la importancia de las redes sociales para incrementar el número de personas beneficiadas por el proyecto de extensión durante el período pandémico.

Palabras clave: Relaciones Comunidad-Institución; Pandemias; Prevención de Enfermedades; Red Social. 


\section{Introdução}

O ano de 2020 está sendo marcado pela histórica pandemia pelo novo Coronavírus (SARS-CoV-2), que se alastrou rapidamente pelo mundo, deixando a população em um grande estado de vulnerabilidade. Esta, em pouco tempo, gerou grandes impactos nos sistemas de saúde, políticos, econômicos e sociais, em quase todo o planeta. Assim, diante do impacto ocasionado pela crise do SARS-CoV-2, causador da COVID-19, o afastamento social se fez necessário a fim de minimizar a propagação da doença1,2,3.

Um dos efeitos observados foi a migração do trabalho presencial para o recurso do teletrabalho (home office), que tem sido amplamente utilizado em diferentes setores. Essa adaptação dos afazeres é uma estratégia para o mercado continuar economicamente ativo, assim, vários órgãos públicos e privados desempenham normalmente as atividades que aconteceriam de forma presencial1,2. Um exemplo é a educação, pois as escolas e universidades migraram suas aulas presenciais para o ambiente virtual, incluindo a continuidade de outras atividades desempenhadas dentro das unidades ${ }^{2}$.

A extensão é uma das atividades realizadas no âmbito universitário, voltada a atender as necessidades da comunidade interna e, principalmente, externa, desenvolvendo ações sociais onde os acadêmicos, com auxílio de professores, elaboram atividades que visam a promoção do bem-estar social. No entanto, diante do cenário atual, emergiu a necessidade de migração para sistema de home office, adaptando também a extensão universitária à nova realidade ${ }^{1,4}$.

Desta forma, o presente artigo tem o objetivo de relatar a experiência de um projeto de extensão universitária que, até o período pré-pandemia, incentivava a adoção de hábitos saudáveis por meio de oficinas presenciais com seu público-alvo: frequentadores de Centros de Arte e Lazer (CAL) e Centros de Referência de ssistência Social (CRAS) mas, com o advento da pandemia, migrou as atividades ara o ambiente virtual, com postagens em redes sociais, mostrando como foram os safios e adaptações para permanecer nesse novo cenário global. 


\section{ciênciála
pural}

\section{Metodologia}

Esse trabalho é um estudo descritivo, do tipo relato de experiência, no qual foi retratada a adaptação do Projeto de extensão universitária: “Educação Alimentar e Nutricional para Promoção da Cidadania, da Saúde e Prevenção de Doenças", vinculado ao curso de Nutrição da Universidade do Vale do Itajaí (UNIVALI), situada em Itajaí-SC. O projeto, vigente há oito anos, tem o objetivo de garantir o acesso a informações e orientações a grupos vulneráveis, de forma contínua, permanente e planejada, por meio da vigilância alimentar e nutricional e de ações educativas no âmbito da alimentação saudável e consequente promoção da cidadania, da saúde e prevenção de doenças. As atividades adaptadas à nova realidade que serão aqui mencionadas ocorreram no primeiro semestre de 2020, iniciando no mês de maio e finalizando em julho.

Após uma reunião de equipe de forma remota, o projeto que até então realizava suas funções somente de forma presencial, teve que se reestruturar para o novo formato, o digital. Foi criado um novo cronograma de atividades, contemplando reuniões semanais, estruturado o design do projeto para as publicações, criadas contas nas redes sociais e selecionados temas relevantes para serem abordados semanalmente, a fim de promover a adequação ao novo cenário global. Os temas abordados em cada postagem para as redes sociais, Facebook® e Instagram ${ }^{\circledR}$, têm o intuito de melhorar a saúde das pessoas, auxiliando-as a terem práticas saudáveis de alimentação e conscientização sobre nutrientes importantes ao sistema imunológico, durante a pandemia. Foram criadas sete postagens, na forma de fotos e textos, cujos assuntos foram: "Chás para a ansiedade", "Alimentos que melhoram o astral", "Intestino saudável", "Temperos saudáveis", "Alimentos que melhoram a imunidade", "Prato saudável" e "Processamento dos alimentos". As postagens para redes sociais foram realizadas uma vez por semana, por dois acadêmicos do curso Nutrição e bolsistas do projeto, tendo sempre a supervisão das professoras sponsáveis. As postagens desenvolvidas tiveram como objetivo de influenciar o lico-alvo do projeto sobre Educação Alimentar e Nutricional (EAN): 
frequentadores de Centros de Arte e Lazer (CAL), Centros de Referência de Assistência Social (CRAS) e demais seguidores das páginas nas redes sociais. O método instituído pela Universidade executora como forma de escriturar o número de pessoas beneficiadas, define quantas pessoas curtiram cada publicação ${ }^{5}$.

\section{Resultados}

Devido ao novo cenário global, algumas atividades de trabalho tiveram que se adaptar a um novo formato, o digital. A extensão universitária, como tantos outros setores da sociedade, foi bastante afetada pela pandemia, pois ações sociais que seriam realizadas em locais estabelecidos para seus públicos alvos, não puderam acontecer. Assim, foi necessário utilizar ferramentas digitais para continuar executando suas tarefas ${ }^{1,5}$. A utilização das redes sociais Facebook ${ }^{\circledR}$ e Instagram $\AA$ ajudaram o projeto a desempenhar suas atividades de forma remota. Foram postados materiais, na forma de fotos e textos, com temas relevantes para a saúde de forma geral e pertinentes para o momento em que estamos enfrentando.

Quadro 1. Relação de postagem para as redes sociais e o número de pessoas atingidas. ItajaíSC, 2020.

\begin{tabular}{|c|c|c|}
\hline TEMAS & REDES SOCIAIS & NÚMERO DE CURTIDAS \\
\hline Chás para a ansiedade & Facebook® e Instagram $\AA$ & 51 \\
\hline $\begin{array}{c}\text { Alimentos que melhoram o } \\
\text { astral }\end{array}$ & Facebook® e Instagram ${ }^{\circledR}$ & 28 \\
\hline Intestino saudável & Facebook® e Instagram $₫$ & 19 \\
\hline Temperos saudáveis & Facebook $₫$ e Instagram $\AA$ & 30 \\
\hline $\begin{array}{l}\text { Alimentos que melhoram a } \\
\text { imunidade }\end{array}$ & Facebook® e Instagram $®$ & 20 \\
\hline Prato saudável & Facebook® e Instagram $\AA$ & 16 \\
\hline Processamento dos alimentos & Facebook® e Instagram ${ }^{\circledR}$ & 20 \\
\hline TOTAL GERAL & - & 184 \\
\hline
\end{tabular}

Legenda: o público beneficiado é representado pelo número de curtidas nas publicações conforme ${ }^{5}$. 


\section{Discussão}

As redes sociais, Facebook® e Instagram $®$ foram criadas nos anos de 2004 e 2010, respectivamente, com o intuito de permitir que o usuário compartilhe e experimente momentos com seus seguidores por meio da publicação de fotos, textos e vídeos ${ }^{6}$. $\mathrm{O}$ Brasil é o quarto país que mais utiliza o Facebook®, com 120 milhões de usuários e o terceiro que mais usa o Instagram $®$, com 69 milhões de usuários. Dessa forma, as duas redes sociais se tornam uma ferramenta importante para divulgação dos materiais elaborados pelo projeto durante o período de isolamento social, pelo alcance do público que é atingido pelas publicações ${ }^{6}$.

A primeira postagem realizada nas redes sociais do projeto foi sobre "Chás para ansiedade", pois, a ansiedade é um problema de saúde que acomete uma grande quantidade de pessoas e que está se agravando em decorrência da pandemia. É um estado emocional com componentes psicológicos e fisiológicos, podendo ser benéfica ou prejudicial à saúde do ser humano. Desta forma, a utilização de chás com ação calmante é uma ótima estratégia para auxiliar na melhora dessa situação ${ }^{6}$. Por isso, foram sugeridos cinco chás: "Camomila", "Alecrim", "Melissa", "Mulungu" e "Passiflora", que desempenham essa ação calmante e que podem ajudar as pessoas durante e após esse período 7,8 .

O objetivo da publicação foi proporcionar ao leitor o conhecimento sobre cada chá mencionando para o manejo da ansiedade, mostrando os benefícios individuais de cada um para a saúde, quantidades de uso diário, tempo de uso e o modo de preparo, que pode ocorrer por infusão, quando as folhas ou flores ficam imersas em água quente por alguns minutos, ou decocção, quando parte das plantas, como o rizoma, são fervidas junto com a água por alguns minutos ${ }^{8,9}$. O material elaborado foi aceito positivamente pelo público alvo e os demais seguidores das redes sociais, o que reflete no número de curtidas. Durante esse período atípico que a população vem frentando, as pessoas estão preocupadas e buscando informações sobre como evenir e aliviar os sintomas de ansiedade ocasionados pelo distanciamento social. 


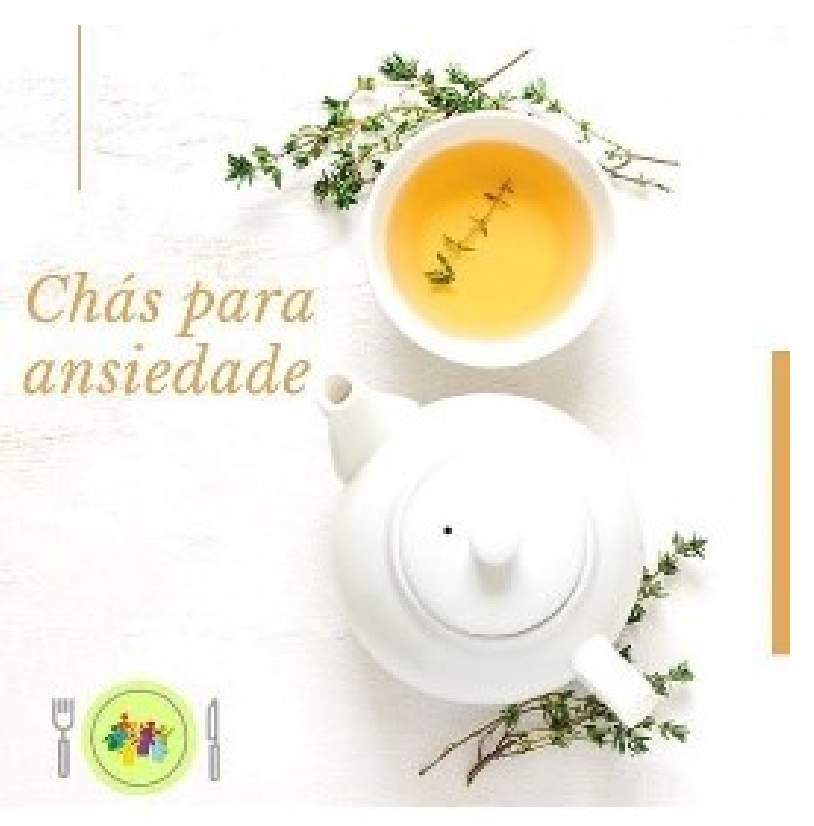

Figura 1. Material elaborado para publicação “Chás para ansiedade”. Itajaí-SC, 2020.

Ainda, fazendo relação com transtornos psicológicos que estão propícios a surgir durante o isolamento social, a segunda publicação intitulava-se "Alimentos que melhoram o astral". A ciência tem apontado que certos alimentos podem melhorar o estado emocional, diminuindo a tristeza, combatendo a depressão e a ansiedade e melhorando o mau humor ${ }^{10}$.

A publicação cita alimentos como banana, ovos, leite e derivados, abacate, peixes, oleaginosas, aveia e cacau são fontes de triptofano e estimulam a produção e liberação de neurotransmissores, substâncias que transmitem impulsos nervosos ao cérebro e são responsáveis pelas sensações de bem-estar e prazer, como a serotonina, dopamina e a noradrenalina ${ }^{10,11}$. Apesar da segunda publicação não ter gerado o mesmo engajamento da primeira, o público-alvo do projeto foi alcançado e a mensagem que aconteceria presencialmente foi transmitida, além de estar disponível para acesso da população. 


\section{Alimentos que melhoram o astral}
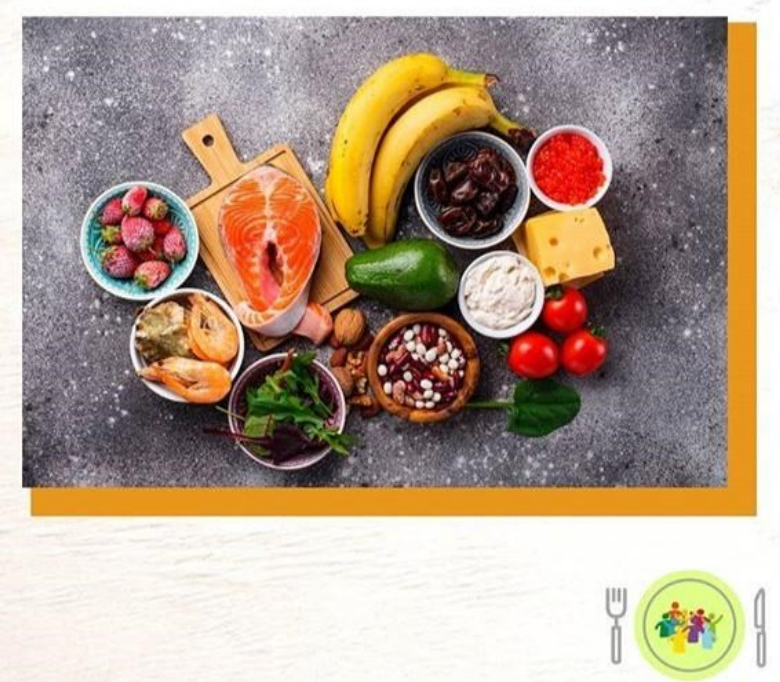

Figura 2. Material elaborado para publicação "Alimentos que melhoram o astral". Itajaí-SC, 2020.

Adotar hábitos saudáveis e uma alimentação natural e equilibrada, é a melhor maneira para manter o intestino funcionando bem, órgão responsável por produzir hormônios do bem-estar, então a terceira postagem intitulava-se: "Intestino saudável", onde foi ressaltado como cuidar do intestino. O consumo de frutas, verduras e cereais integrais é indispensável para fornecer um adequado aporte de fibras, que são essenciais para a saúde intestinal. Além das fibras, são necessários também outros componentes, como probióticos, que são alimentos que contêm micro-organismos benéficos para o corpo. São exemplos de alimentos probióticos: os fermentados como o chucrute, kimchee, kombucha e kefir. Ainda, ressalta-se a necessidade de uma adequada ingestão hídrica, pois auxilia no movimento peristáltico e na evacuação ${ }^{12}$.

A terceira postagem foi uma das que teve menor engajamento, isso se deu devido à dificuldade que o projeto teve durante as publicações de verificar o horário de pico de acesso dos seguidores, sendo esse um dos motivos da oscilação do número curtidas. Outra questão levantada pelos membros do projeto foi que o assunto stava bastante em alta e acabou se tornando repetitivo, porém é relevante ser ordado devido às funções que o intestino tem para a saúde da população. 


\section{ciênncia
pural}

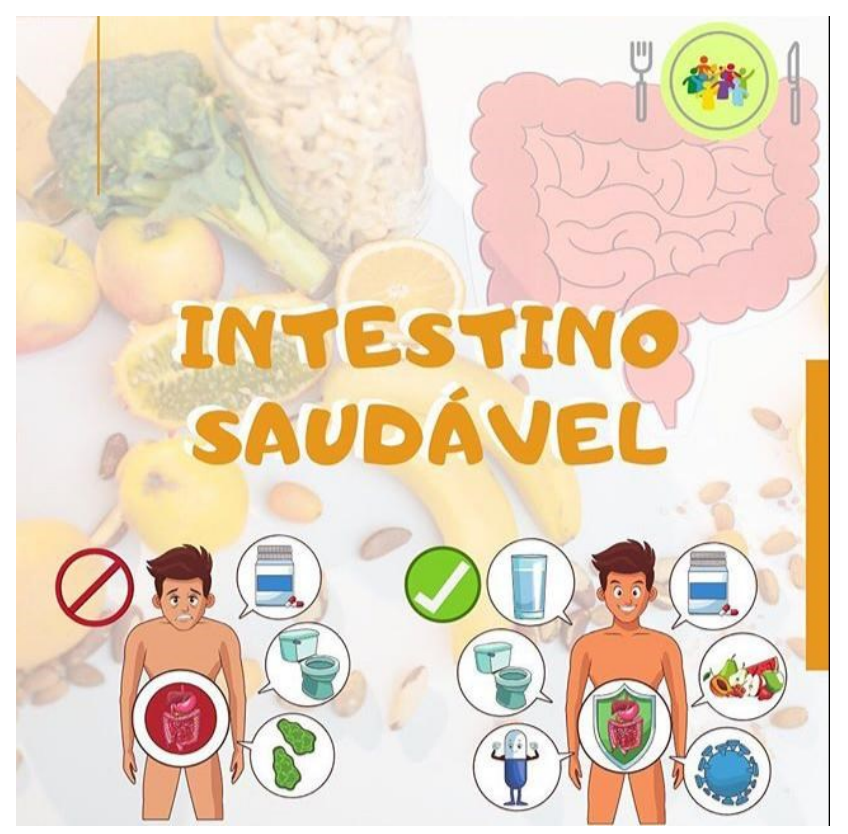

Figura 3. Material elaborado para publicação

"Intestino Saudável". Itajaí-SC, 2020.

Em tempos de pandemia, onde as refeições são produzidas em casa, é fundamental utilizar temperos naturais ao invés dos industrializados, tema da quarta postagem, "Temperos saudáveis", foi realizada pensando nisso. Os temperos são determinantes para preparar um prato saboroso e até mesmo inesquecível11. E, para isso, não é preciso abusar do sal. Afinal, os temperos naturais, como alecrim, alho, cebola, coentro, cúrcuma, manjericão, salsinha e sálvia, possuem ações antiinflamatórias, antioxidantes, calmantes, estimulantes, cicatrizantes e antibióticas. Uma vantagem que existe entre os temperos e especiarias é que eles são muito versáteis e podem ser utilizados em vários tipos de receitas ${ }^{13,14}$.

A quarta postagem surpreendeu devido ao engajamento que gerou, traduzido no número de curtidas. Esse parâmetro é avaliado quando a publicação alcança mais curtidas em comparação a anterior e está entre as mais acessadas ${ }^{6}$. Isso mostra o interesse por temperos saudáveis para preparo das refeições. Outra questão levantada oi a predominância feminina entre os seguidores, o que reflete no alcance da blicação, pois habitualmente as mulheres cozinham para seus familiares. 


\section{ciênncia
pural}

\section{TEMPEROS 2 SAUDÁVEIS}

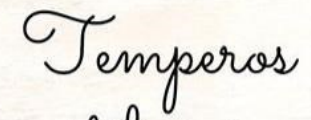

artificiais, nunca mais!
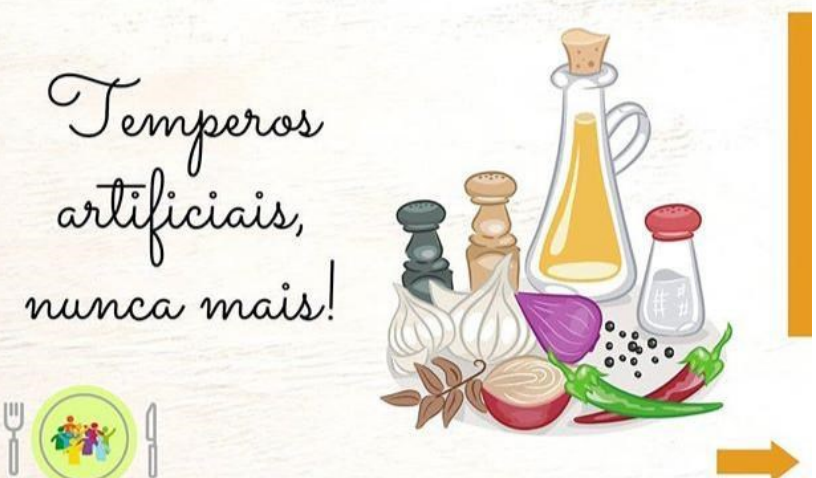

Figura 4. Material elaborado para publicação

“Temperos saudáveis”. Itajaí-SC, 2020.

O fortalecimento do sistema imunológico é fundamental para o combate de qualquer tipo de contaminação, seja ela bacteriana, fúngica ou viral. Mas para isso é necessário adotar um estilo de vida saudável ${ }^{15}$. A quinta postagem abordou sobre "Alimentos que melhoram imunidade", onde foi descrito sobre alimentos antioxidantes, como os ricos em vitamina $\mathrm{C}$, que beneficiam as células de defesa do organismo ao combate de patógenos ${ }^{16,17}$. Posteriormente, foram apresentadas mais duas publicações sobre como montar um prato saudável e sobre o grau de processamento dos alimentos, os quais são assuntos relevantes para a incorporação de hábitos mais saudáveis ${ }^{15}$. Apesar do engajamento ter diminuído durante as três últimas publicações, todos os assuntos abordados estão relacionados com a melhora da saúde e consequente do bem-estar da população, por meio da ingestão de alimentos saudáveis e saborosos ${ }^{13,16}$.

\section{Tonclusões}

A crise do novo SARS-CoV-2 tem impactado diversos setores, dentre eles o da ducação, no qual as atividades realizadas dentro da universidade estão sendo anejadas e readaptadas para o modelo on-line, dentre elas a extensão universitária. 


\section{ciência
purál}

O projeto de extensão “Educação Alimentar e Nutricional na Promoção da Cidadania, da Saúde e Prevenção de Doenças", que normalmente realizava ações sociais de EAN para a comunidade em vulnerabilidade social de Itajaí-SC, teve que se adaptar ao novo formato com publicações para as redes sociais, Facebook® e Instagram ${ }^{\circledR}$.

Apesar das dificuldades iniciais de planejamento de cronograma, temas abordados e o alcance do público alvo, conseguimos reestruturar bem o projeto ao novo formato. Tivemos dificuldades em encontrar o horário de pico de acesso dos seguidores e de diversificar temas que já estavam sendo muito divulgados nas redes sociais, o que refletiu nas oscilações das curtidas das publicações. Porém, conseguimos perceber, com os retornos que tivemos, a capacidade de influenciar positivamente a vida das pessoas, buscando trazer assuntos pertinentes e com embasamento científico, para assim, mesmo que remotamente, tentar melhorar a qualidade de vida da população. Destacamos a importância das redes sociais para o aumento do número de pessoas beneficiadas pelo projeto de extensão durante o período pandêmico.

\section{Referências}

1. Wu Z, McGoogan JM. Characteristics of and Important Lessons From the Coronavirus Disease 2019 (COVID-19) Outbreak in China: Summary of a Report of 72314 Cases From the Chinese Center for Disease Control and Prevention. JAMA. 2020; 323(13): 1239-1242.

2. Castro BLG, et al. COVID-19 e organizações: estratégias de enfrentamento para redução de impactos. Rev de Psicologia 2020; 20(3): 1059-1063.

3. Teixeira SIN, Barros de Oliveira LM, dos Santos IR, Ferreira Pinheiro JM, Dantas de Araújo Oliveira MR, Dantas de Lira NR et al. Procedimento operacional padrão na assistência nutricional ao paciente com covid 19: relato de experiência. Rev Ciênc Plural 2020; 6(2): 156-69.

4. Oliveira EE. Um auto relato sobre a participação no projeto "Ateliê do sorriso": um minho percorrido da extensão à sociedade. Rev Ciência Plural 2019; 5(3): 72-88.

Kuhn IN, Remi AD. Elaboração e acompanhamento de projetos públicos e sociais. 1 Editora Unijuí: Rio Grande do Sul 2008. 
6. Coelho RLF, Oliveira DS, Almeida MIS. Does social media matter for post typology? Impact of post content on Facebook and Instagram metrics. Emerald Insight 2016; 40(4): 458-471.

7. Brooks SK, Webster RK; Smith LE; Woodland L; Wessely S; Greenberg N et al. The psychological impact of quarantine and how to reduce it: Rapid review of the evidence. The Lancet. 2020; 395(10227): 912-920.

8. Brasil. Programa Nacional de Plantas Medicinais e Fitoterápicos. Ministério da Saúde: Brasília 2016.

9. Henn R, Almeida EB, Guedes MR, Santos ACC, Ott APC, Vargas AJ, et al. Educação alimentar e nutricional para grupos vulneráveis: um relato de experiência das vivências de extensão universitária. Rev Bras Tecnologias Sociais 2018; 5(1): 59-68.

10. Testoni Chaves Pereira NC, Hüther da Costa K, Kaio Silva Nunes R, Larissa Borges P, do Nascimento Albuquerque I, Barth Almeida E et al. Ações de educação alimentar e nutricional com grupos em vulnerabilidade social: relato de experiência. Rev Ciência Plural 2020; 6(2): 170-91.

11. Celiberto LS, Franziska AG, Genelle RH, Else SB, Kevan J, Laura MS et al. Inflammatory bowel disease and immunonutrition: novel therapeutic approaches through modulation of diet and the gut microbiome. Immunology 2018; 155(1): 36-52.

12. Brasil. Guia Alimentar da População Brasileira. 2ed. Ministério da Saúde: Brasília 2014.

13. Yahfoufi N, Nawal A, Majed J, Chantal M. The Immunomodulatory and Antiinflammatory Role of Polyphenols. Nutrients MDPI 2018; 10(11): 1618.

14. Pae M, Wu D. Nutritional modulation of age-related changes in the imune system and risk of infection. Nutrition Research 2017; 41: 14-35.

15. Wu D, Erin DL, Munyong P, Simin NM. Nutritional Modulation of Immune Function: Analysis of Evidence, Mechanisms, and Clinical Relevance. Front Immunoly Journal 2019; 9:3160.

16. Silva FM, Gonçalves LG, Figueiredo RC, Barreto SM, Consumo de alimentos ultraprocessados, IMC e circunferência da cintura no ELSA-Brasil. X Congresso Brasileiro de Epidemiologia 2017. Campinas: Gaolá; 2018. 